\title{
ON THE FORMULATION OF CONSTITUTIVE EQUATIONS FOR LIVING SOFT TISSUES*
}

\author{
By WILLIAM PRAGER ${ }^{1}$ (University of California, San Diego)
}

\begin{abstract}
Soft living tissues deform freely under negligible stresses until a certain strain level is reached at which their stiffness increases sharply. Constitutive equations are developed that describe this kind of mechanical behavior and include Hooke's law as a limiting case. It is shown that, similar to Hooke's law, these constitutive equations assure uniqueness of solution for a broad class of boundary value problems. Possible extensions of the theory are briefly indicated.
\end{abstract}

1. Introduction. The most striking feature of the mechanical behavior of soft living tissues is the occurence of significant strains before measurable stresses develop. Fig. 1 shows the results of a tension test performed by Fung [1] on a specimen excised from the mesentery membrane of a rabbit. It is seen that measurable stresses develop only after the specimen has been elongated to slightly more than twice its relaxed length. From this point on, however, stresses increase rapidly with further elongation.

To some extent, this behavior resembles that stipulated in the theory of ideal locking materials [2]. This remark raises the question whether this theory cannot be modified to correspond more closely to the type of mechanical behavior that is illustrated by Fig. 1. For infinitesimal strains, a simple modification of this kind is explored in this paper.

As is clearly indicated by Fig. 1, the restriction to infinitesimal strains is not realistic for soft living tissures. It appears nevertheless worthwhile to explore the new concepts (and their more immediate consequences) within the domain of infinitesimal strains before attempting to extend them to the realm of finite strains. By itself, this extension should not present insurmontable difficulties, though it will, of course, greatly complicate the solution of specific problems.

2. Models of locking materials. Two types of locking materials have been investigated in the literature. Their mechanical behavior is represented by the following simple models. A particle with the position vector $\mathbf{x}$ is restricted to a plane, strictly convex domain $D$, which is bounded by a smooth, rigid frame. When the particle is not in contact with the frame, it is either free (ideal locking material [2]) or experiencing the elastic restoring force $-c \mathbf{x}$ (elastic, perfectly locking material [3], [4]). When the particle is in contact with the frame, it experiences, in addition, a reaction from the frame. Since there is to be no friction between particle and frame, this reaction has the direction of the interior normal to the frame at the point of contact. When the components of the position vector of the particle are interpreted as typical strain components, and the components of the resultant force acting on the particle as the corresponding stress components, these models respectively represent the mechanical behavior of ideal locking materials and elastic, perfectly locking materials.

\footnotetext{
*Received March 4, 1968. This research was supported by the U. S. Air Force Office of Scientific Research through Grant AF-AFOSR-1186-67.

${ }^{1}$ Now at Brown University, Previdence, R. I.
} 


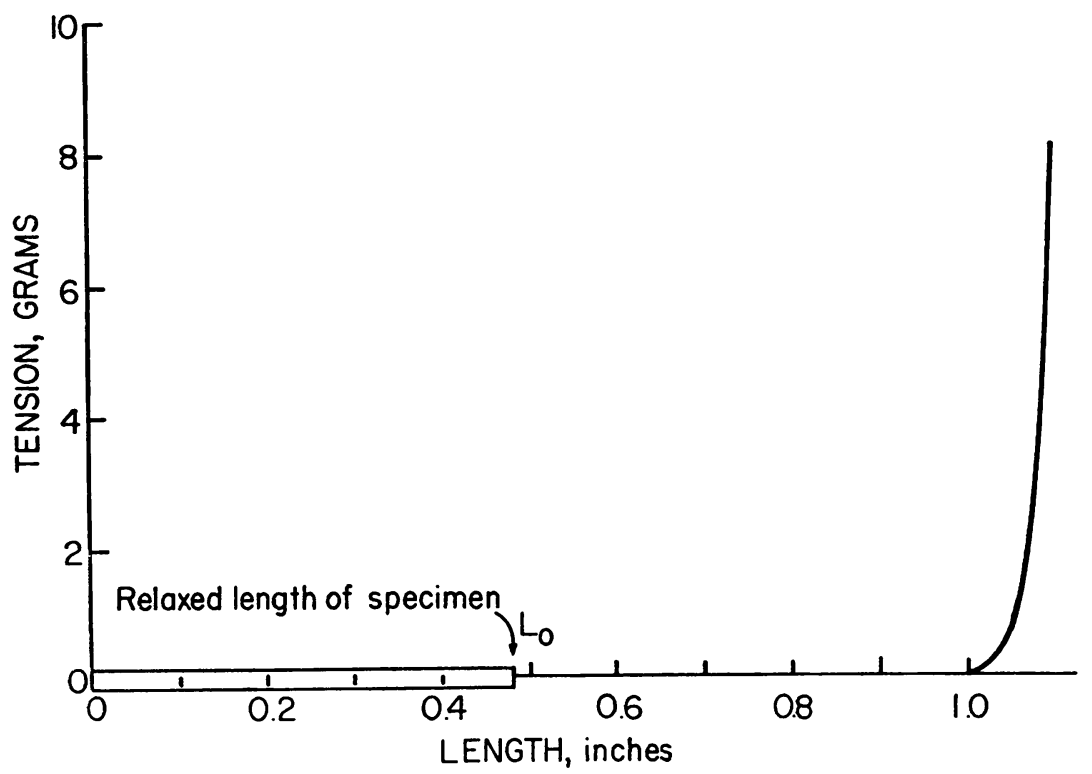

FIG. 1: Tension of strip of rabbit mesentery (according to Fung [1]).

To represent the behavior illustrated by Fig. 1, the model of the ideal locking material may be modified as follows: the rigid frame is removed, and the exterior of the convex domain $D$ is filled with a substance into which the particle can penetrate against some elastic resistance, whereas it can move freely inside the domain $D$.

3. Constitutive equation. The first step towards a constitutive equation for the mechanical behavior described by the new model is the specification of the "free" domain $D$, that is the specification of the states of strain that are associated with negligible stresses.

The infinitesimal strain tensor will be denoted by $\epsilon_{i j}$, where the subscripts $i$ and $j$ have the range $1,2,3$. The free domain $D$ will be assumed to correspond to nonpositive values of some function $f\left(\epsilon_{11}, \epsilon_{12}, \epsilon_{13}, \epsilon_{21}, \epsilon_{22}, \epsilon_{23}, \epsilon_{31}, \epsilon_{32}, \epsilon_{33}\right)$, which will be abbreviated by $f(\epsilon)$. Note that symmetric components of strain, such as $\epsilon_{12}$ and $\epsilon_{21}$, are formally treated as independent arguments of the function $f$, which is supposed to be symmetric in these components. Moreover, $f$ will be assumed to be a convex function of its arguments in the sense that

$$
\left(\epsilon_{i j}-\epsilon_{i j}^{*}\right) \partial f / \partial \epsilon_{i i} \geq f(\epsilon)-f\left(\epsilon^{*}\right),
$$

where $\epsilon_{i j}$ and $\epsilon_{i j}^{*}$ are any two states of strain and a repeated letter subscript indicates summation over the range 1, 2, 3 of this subscript. The derivatives of $f$ in (1) are to be evaluated for the state $\epsilon_{i j}$, and the equality sign is to hold only if $\epsilon_{i j}=\epsilon_{i j}^{*}$ (strict convexity).

The general role of convexity assumptions of this type in solid mechanics has been stressed by Hill [5]. In the present context, the convexity of the free domain $D$ in strain space directly follows from the assumed convexity of the function $f$. Indeed, since this domain is to correspond to nonpositive values of $f$, its boundary $B$ has the equation $f=0$. If the states of strain $\epsilon_{i j}$ and $\epsilon_{i j}^{*}$ are respectively represented by points $P$ and $P^{*}$ 
on this boundary, $f(\epsilon)=f\left(\epsilon^{*}\right)=0$ and (1) reduces to

$$
\left(\epsilon_{i j}-\epsilon_{i i}^{*}\right) \partial f / \partial \epsilon_{i i} \geq 0 .
$$

This inequality states that the vector from $P^{*}$ to $P$ cannot make an acute angle with the direction of the exterior normal to $B$ at $P$, and thus expresses the convexity of $B$.

The simplest constitutive equation with the required properties is

$$
\sigma_{i j}=2\langle f\rangle \partial f / \partial \epsilon_{i i},
$$

where $\sigma_{i j}$ is the stress tensor and

$$
\begin{aligned}
\langle f\rangle=0 & \text { if } \quad f<0, \\
=f & \text { if } \quad f \geq 0 .
\end{aligned}
$$

Note that Hooke's law is obtained as a special case of (2) when $f$ is taken as

$$
f=\bar{f}=\left\{\frac{1}{2}\left(\lambda \epsilon_{i i} \epsilon_{i i}+2 \mu \epsilon_{i i} \epsilon_{i j}\right)\right\}^{1 / 2},
$$

where $\lambda$ and $\mu$ are Lamé's constants. Indeed, the radicand in (5) is the strain energy $U$, which is positive for nonvanishing strain. Accordingly, the angle brackets can be omitted in (3) and this constitutive equation reduces to

$$
\sigma_{i j}=\partial\left(\bar{f}^{2}\right) / \partial \epsilon_{i j}=\partial U / \partial \epsilon_{i j} .
$$

Returning now to the general discussion of the constitutive equation (2), note that the rate at which the stress $\sigma_{i j}$ does work on a strain increment $d \epsilon_{i j}$ is given by

$$
\begin{aligned}
\sigma_{i j} d \epsilon_{i i}=2\langle f\rangle d f & =0 \quad \text { for } f<0, \\
& =d\left(f^{2}\right) \text { for } f \geq 0 .
\end{aligned}
$$

It follows that the constitutive equation (3) defines a material that is elastic in the sense that the total work for any closed cycle of strain is zero.

Next to (5), which leads to Hooke's law, the most obvious choice of $f$ is

$$
f=\bar{f}-k,
$$

where $\bar{f}$ is given by (5). From (3) and (8), it follows that

$$
\sigma_{i j}=\langle\bar{f}-k\rangle\left\{\left(\lambda \epsilon_{k k} \delta_{i j}+2 \mu \epsilon_{i j}\right) / \bar{f}\right\} .
$$

Here, the expression in braces may be regarded as a normalized Hooke stress for the strain $\epsilon_{i j}$, while $\langle\bar{f}-k\rangle$ is a cut-off factor, which reduces this normalized Hooke stress to zero whenever $\bar{f} \leq k$. For $k=0$, the constitutive equation (9) reduces to Hooke's law.

4. Uniqueness. As is well known, Hooke's law assures uniqueness of solution for the basic boundary value problems of the classical theory of elasticity. It will be shown in this section that a similar statement can be made for the constitutive law (3).

The following boundary value problem will be considered. A material with the constitutive equation (3) occupies the simply connected volume $V$ with the regular surface $S$. The body force $F_{i}$ (per unit volume) is given throughout $V$; on the part $S_{T}$ of the surface $S$, the surface traction $T_{i}$ is prescribed, and on the remainder $S_{U}$ of $S$, the (infinitesimal) surface displacement $u_{i}$ is given. The prescribed surface displacements are not supposed to be compatible with a rigid body motion of the material in $V$.

To investigate the extent to which these data uniquely determine the stress field 
$\sigma_{i}$ and the displacement field $u_{i}$ in $V$, assume that the boundary value problem admits two solutions with the displacement, strain, and stress fields $u_{i}, \epsilon_{i j}, \sigma_{i j}$ and $u_{i}^{*}, \epsilon_{i j}^{*}, \sigma_{i j}^{*}$. Since each stress field satisfies the equations of equilibrium in $V$ and the boundary conditions on $S_{T}$, and since each displacement field satisfies the boundary conditions on $S_{U}$, it follows from the principle of virtual work that

$$
\int\left(\sigma_{i i}-\sigma_{i j}^{*}\right)\left(\epsilon_{i j}-\epsilon_{i i}^{*}\right) d V=0 .
$$

To prove uniqueness, we show that the integrand

$$
I=\left(\sigma_{i i}-\sigma_{i j}^{*}\right)\left(\epsilon_{i j}-\epsilon_{i j}^{*}\right)
$$

in (10) is positive unless $\epsilon_{i i}=\epsilon_{i j}^{*}$.

According to the constitutive equation (3), we have

$$
I=2\left(\epsilon_{i j}-\epsilon_{i j}^{*}\right)\left\{\langle f\rangle \partial f / \partial \epsilon_{i i}-\left\langle f^{*}\right\rangle \partial f / \partial \epsilon_{i j}^{*}\right\},
$$

where $f=f(\epsilon)$ and $f^{*}=f\left(\epsilon^{*}\right)$. Using (1) and the relation obtained from it by interchanging starred and unstarred quantities, we obtain

$$
I \geq 2\left(f-f^{*}\right)\left(\langle f\rangle-\left\langle f^{*}\right\rangle\right),
$$

where the equality sign holds only for $\epsilon_{i i}=\epsilon_{i i}^{*}$.

In discussing (13), we assume at first that $\epsilon_{i j} \neq \epsilon_{i j}^{*}$ and hence use (13) as strict inequality. The following cases may arise at the point where $I$ is to be evaluated:

(a) $f>0, f^{*}>0$ : neither one of the solutions predicts vanishing stress, and (13) reduces to

$$
I>2\left(f-f^{*}\right)^{2} \geq 0
$$

(b) $f>0, f^{*}<0$ : only the starred solution predicts vanishing stress, and (13) takes the form

$$
I>2\left(f-f^{*}\right) f>0
$$

(c) $f \leq 0, f^{*}>0$ : can be shown to furnish $I>0$ by the same lind of argument as under (b);

(d) $f \leq 0, f^{*} \leq 0$ : both solutions predict vanishing stress, that is, $\sigma_{i i}=\sigma_{i j}^{*}=0$ and hence $I=0$.

It follows from this discussion that, to satisfy (10), we must either have case (d) throughout $V$ or abandon the assumption that $\epsilon_{i j} \neq \epsilon_{i j}^{*}$. In either case we have $\sigma_{i j}=\sigma_{i j}^{*}$ throughout $V$.

The considered boundary value problem thus defines a unique stress field. The strain field obviously need not be unique where both solutions predict vanishing stresses, but elsewhere it is, in fact, unique.

5. Generalization and conclusion. For brevity, only a single, though fairly general, type of boundary value problem has been investigated. The uniqueness proof, however, is readily extended to the other boundary value problems considered in the classical theory of elasticity. For example, only the shearing components of the surface traction may be given on $S_{T}$ together with the normal component of the displacement.

Another manner in which the present investigation could be extended is the use of 
the inequality (1) to establish extremum characterizations of the solution of the considered boundary value problem.

Finally, more general constitutive equations could be discussed, for which the free domain $D$ is specified by a set of functions $f_{1}(\epsilon), f_{2}(\epsilon), \cdots, f_{n}(\epsilon)$, all of which must be nonpositive if the considered state of strain is to be associated with negligible strains.

The fact that a comprehensive and consistent theory can be developed in this manner for infinitesimal strains is encouraging and therefore seemed worth reporting. It should be kept in mind, however, that this theory will have to be extended to finite strains before it can become useful in treating the deformations of soft living tissues.

\section{REFERENCES}

[1] Y. C. B. Fung, The elasticity of soft tissues in simple elongation, Amer. J. Physiology 213, 1532-1544 (1967), Figure 2

[2] W. Prager, On ideal locking materials, Trans. Soc. Rheology 1, 169-175 (1957)

[3] W. Prager, Unilateral constraints in mechanics of continua, Atti del Simposio, Lagrangiano, Accad. del Scienze di Torino (1964) pp. 181-191

[4] W. Prager, On elastic perfectly locking materials, Proc. 11th International Congress Appl. Mech., Munich, 1964 Springer; Berlin, 1966, pp. 538-544

[5] R. Hill, New horizons in the mechanics of solids, J. Mech. Phys. Solids 5, 66-74 (1956) 